\title{
Does training level affect the accuracy of visual assessment of capillary refill time?
}

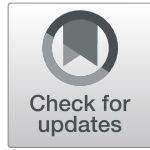

\author{
Koichiro Shinozaki ${ }^{1,2^{*}}$ D, Lee S. Jacobson ${ }^{2}$, Kota Saeki ${ }^{3}$, Naoki Kobayashi ${ }^{4}$, Steve Weisner ${ }^{3}$, Julianne M. Falotico ${ }^{2}$, \\ Timmy Li ${ }^{2}$, Junhwan Kim', Joshua W. Lampe ${ }^{1,5}$ and Lance B. Becker ${ }^{1,2}$
}

Capillary refill time (CRT) measured at the bedside is widely promulgated in critical care and intensive care medicine $[1,2]$. However, traditional CRT measurements are relatively subjective [3], and the accuracy is questionable given that clinicians use the naked eye to perform these visual assessments $[4,5]$. The purpose of our study was to evaluate the accuracy of visually assessed CRT among observers who have different training levels.

Fingernail compression and release videos were recorded from patients in the emergency department (ED) at a suburban, quaternary care teaching hospital in New York. We used our image analysis software to analyze the corresponding fingernail video to calculate patient's CRT (Fig. 1). Nine clinicians and two non-clinicians voluntarily participated as observers to review the videos. Videos from 20 patients were displayed on a screen three times in random order, for a total of 60 videos. The observers watched each fingernail video and pressed a time switch when they deemed the fingernail color had returned to its baseline state. The truth of visually assessed CRT was evaluated by using a correlation of the numbers between the image analysis and the visual assessment. We also sought to determine the intra-observer reliability to evaluate the precision of visual assessments.
Image analysis of CRT of $20 \mathrm{ED}$ patients ranged from 0.47 to $7.98 \mathrm{~s}$, with a mean of $2.44 \pm 2.09 \mathrm{~s}$. The highest intra-observer reliability among the three visual assessment times was displayed by one of the physician assistants $(0.70$ for single measure and 0.88 for average measures); however, it was also as low as 0.15 for a single measure and 0.34 for average measures by one of the non-clinicians. Intra-observer reliability was the highest in attending physicians and physician assistants, followed by residents, nurses, and non-clinicians. The mean intra-observer reliability of the clinicians was higher than the non-clinicians $(0.46$ vs. $0.25, p<0.05)$. Figure 2 shows intra-observer reliability of the video assessment as a function of correlation coefficient of video CRT assessment with image CRT analysis. Observers, who showed a higher correlation with image CRT analysis, demonstrated higher intra-observer reliability, and there was a strong correlation between these coefficient values $(r=0.72, p<$ 0.05).

Visual assessment of CRT is variable. Personal work experience may help improve both truth and precision of CRT assessments and increase the accuracy among individual observers. Therefore, training level appears to be an important factor that affects the reliability of visual CRT assessment.

Full list of author information is available at the end of the article 

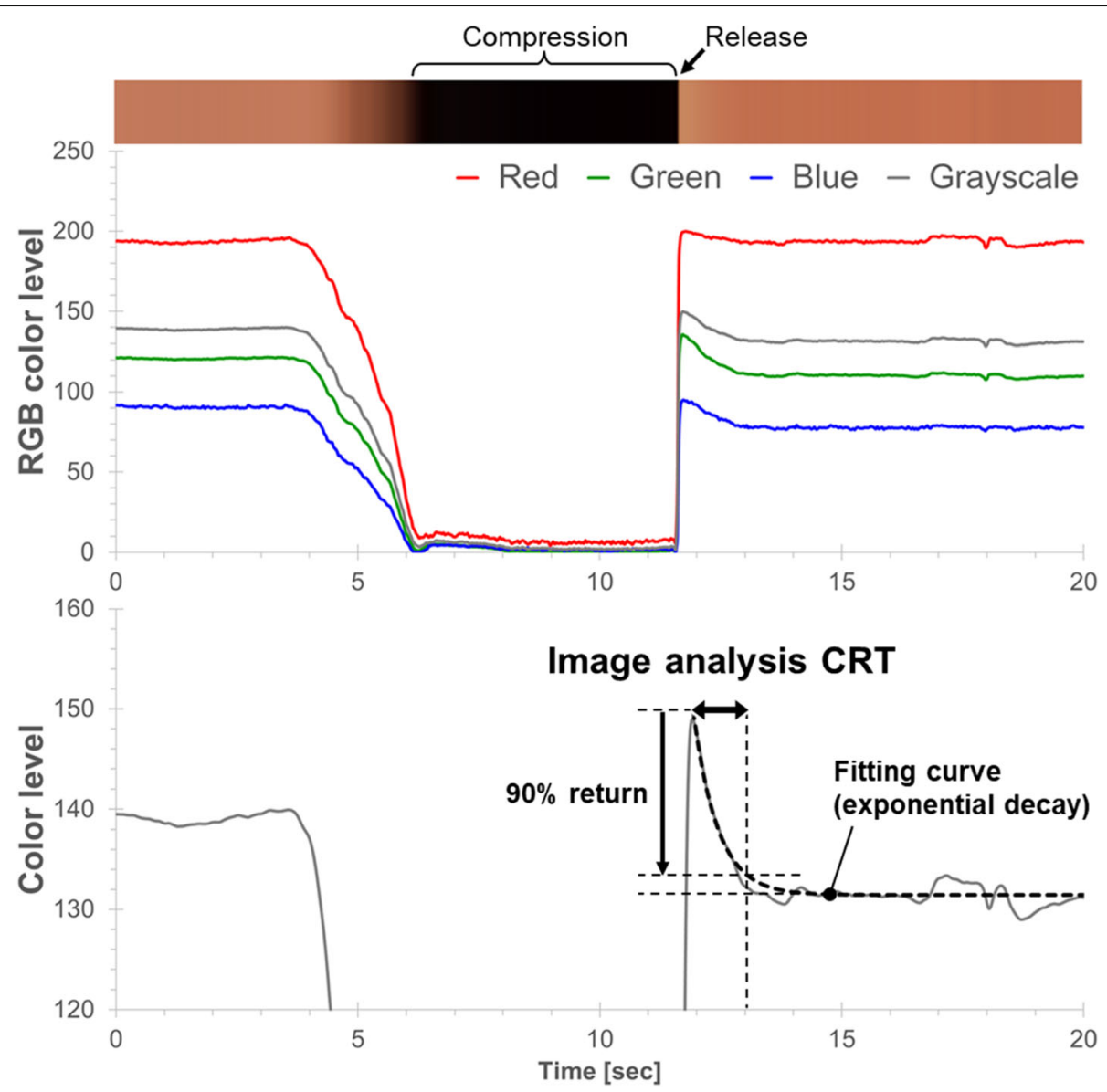

Fig. 1 Image analysis CRT. The recorded videos were used thereafter in a separate setting to calculate CRT via image software analysis (image analysis (RT). Averaged color of the fingernail area was extracted from the digital video file and the color change was represented as RGB waveforms. And then, the RGB waveforms were converted to a grayscale waveform. A curve fitting the returning phase of the grayscale waveform was modeled as an exponential decay using the least squares method. The time to achieve $90 \%$ return of fitting curve was reported as "image analysis CRT" 


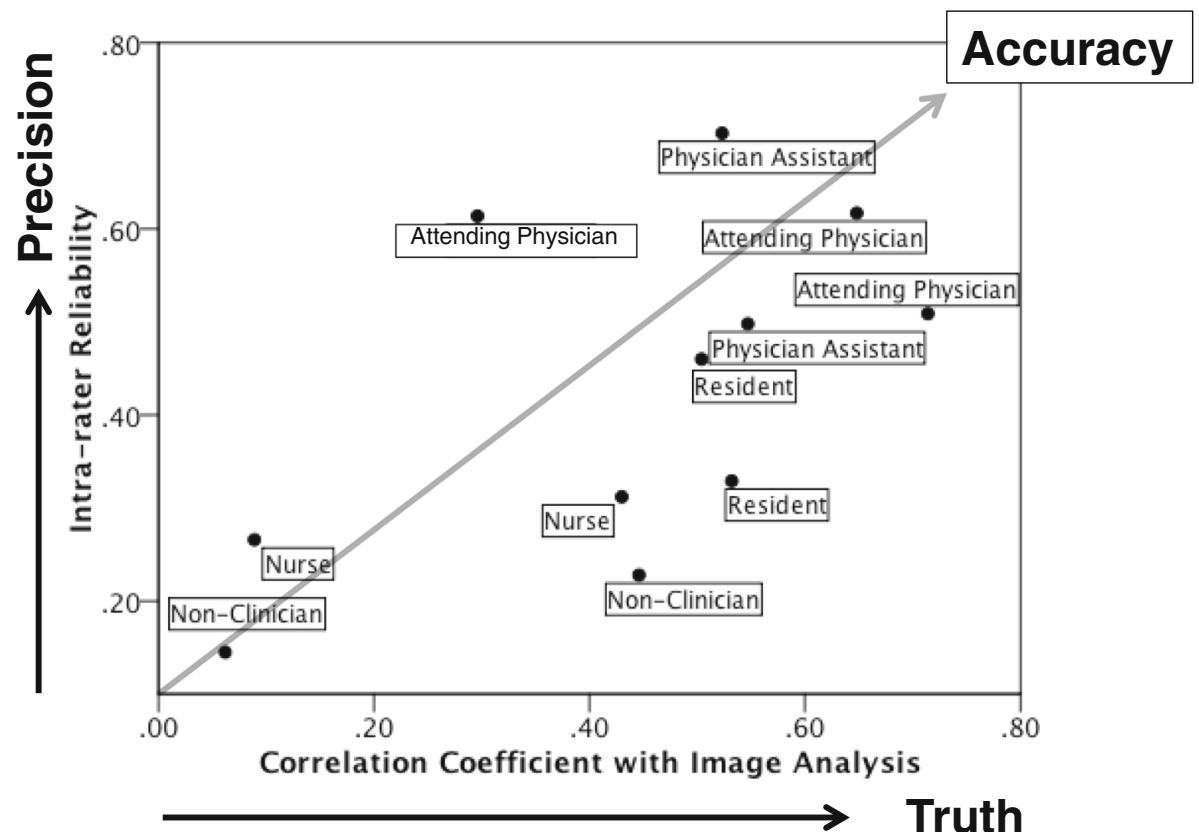

Fig. 2 Scatter plot showing intra-rater (observer) reliability of video assessment CRT as a function of correlation coefficient of video assessment CRT with image analysis CRT. Attending physicians (22 and 28 years of ED work experience), residents (3 years of ED work experience), nurses (2 years of ED work experience), and physician assistants ( 1 and 2 years of ED work experience) participated in the study. Six clinicians were actively performing CRT assessments in their clinical work. Observers, who showed higher correlation with image analysis CRT, demonstrated higher intrarater reliability, and there was a strong correlation between these coefficient values $(r=0.72, p<0.05)$

\section{Acknowledgements}

None.

\section{Funding}

Research reported in this publication was supported by the research grant of Nihon Kohden Corporation.

\section{Availability of data and materials}

The de-identified dataset is held by the corresponding author and the sponsor, and data may be made available in part for secondary analysis by third parties, and access will be considered on a case-by-case basis under our corporate policy.

\section{Authors' contributions}

K. Shinozaki, K. Saeki, and LBB designed the conception of the study; K. Shinozaki, LSJ and K. Saeki performed the acquisition of data; K. Shinozaki analyzed the data; all authors made interpretations of data; K. Shinozaki drafted and JMF edited the manuscript; all authors added intellectual content of revisions to the paper and gave final approval of the version to be published; LBB supervised and enabled the study project.

\section{Ethics approval and consent to participate}

The study protocol was approved by the Institutional Review Board (no. 170805). Informed consent for participation was obtained from all patients prior to the completion of any study procedures.

\section{Consent for publication}

Not applicable.

\section{Competing interests}

LSJ, JMF, TL, and JK have no known conflicts of interest associated with this study, and there has been no significant financial support for this work that could have influenced its outcome. Kota S., NK, and SW are employees of Nihon Kohden Corporation and Nihon Kohden Innovation Center, INC. There are no products in market to declare. This does not alter the authors' adherence to all the journal's policies on sharing data and materials. Koichiro S. and LBB have a patent right of metabolic measurements in critically ill patients. Koichiro S. has a grant/research support from Nihon Kohden Corp.. LBB has a grant/research support from Philips Healthcare, the NIH, Nihon Kohden Corp., Zoll Medical Corp, PCORI, BrainCool, and United Therapeutics and owes patents including 7 issued patents and several pending patents involving the use of medical slurries as human coolant devices to create slurries, reperfusion cocktails, and measurement of respiratory quotient.

\section{Publisher's Note}

Springer Nature remains neutral with regard to jurisdictional claims in published maps and institutional affiliations.

\section{Author details}

${ }^{1}$ The Feinstein Institute for Medical Research, Northwell Health System, 350 Community Dr., Manhasset, NY 11030, USA. ${ }^{2}$ Department of Emergency Medicine, North Shore University Hospital, Northwell Health System, Manhasset, NY, USA. ${ }^{3}$ Nihon Kohden Innovation Center, Cambridge, MA, USA. ${ }^{4}$ Nihon Kohden Corporation, Tokyo, Japan. ${ }^{5}$ ZOLL Medical, Chelmsford, MA, USA.

Received: 2 April 2019 Accepted: 15 April 2019

Published online: 06 May 2019

\section{References}

1. Van Genderen ME, Paauwe J, de Jonge J, van der Valk RJ, Lima A, Bakker J, van Bommel J. Clinical assessment of peripheral perfusion to predict postoperative complications after major abdominal surgery early: a prospective observational study in adults. Crit Care. 2014;18:R114.

2. Morimura N, Takahashi K, Doi T, Ohnuki T, Sakamoto T, Uchida Y, Takahashi $\mathrm{H}$, et al. A pilot study of quantitative capillary refill time to identify high blood lactate levels in critically ill patients. Emerg Med J. 2015;32:444-8.

3. Kawaguchi R, Nakada TA, Oshima T, Shinozaki M, Nakaguchi T, Haneishi H, Oda S. Optimal pressing strength and time for capillary refilling time. Crit Care. 2019;23:4. 
4. Alsma J, van Saase JLCM, Nanayakkara PWB, Schouten WEMI, Baten A, Bauer MP, Holleman F, et al. The power of flash mob research: conducting a nationwide observational clinical study on capillary refill time in a single day. Chest. 2017:151:1106-13.

5. Pickard A, Karlen W, Ansermino JM. Capillary refill time: is it still a useful clinical sign? Anesth Analg. 2011;113:120-3. 